\title{
Anisotropic particle in viscous shear flow: Navier slip, reciprocal symmetry, and Jeffery orbit
}

\author{
Jiaolong Zhang \\ Nano Science and Technology (NSNT) Program, Hong Kong University of Science and Technology, Clear Water Bay, Kowloon, Hong Kong \\ Xinpeng $\mathrm{Xu}$ and Tiezheng Qian* \\ Department of Mathematics, Hong Kong University of Science and Technology, Clear Water Bay, Kowloon, Hong Kong
}

(Received 20 January 2015; published 26 March 2015)

\begin{abstract}
The hydrodynamic reciprocal theorem for Stokes flows is generalized to incorporate the Navier slip boundary condition, which can be derived from Onsager's variational principle of least energy dissipation. The hydrodynamic reciprocal relations and the Jeffery orbit, both of which arise from the motion of a slippery anisotropic particle in a simple viscous shear flow, are investigated theoretically and numerically using the fluid particle dynamics method [Phys. Rev. Lett. 85, 1338 (2000)]. For a slippery elliptical particle in a linear shear flow, the hydrodynamic reciprocal relations between the rotational torque and the shear stress are studied and related to the Jeffery orbit, showing that the boundary slip can effectively enhance the anisotropy of the particle. Physically, by replacing the no-slip boundary condition with the Navier slip condition at the particle surface, the cross coupling between the rotational torque and the shear stress is enhanced, as manifested through a dimensionless parameter in both of the hydrodynamic reciprocal relations and the Jeffery orbit. In addition, simulations for a circular particle patterned with portions of no-slip and Navier slip are carried out, showing that the particle possesses an effective anisotropy and follows the Jeffery orbit as well. This effective anisotropy can be tuned by changing the ratio of no-slip portion to slip potion. The connection of the present work to nematic liquid crystals' constitutive relations is discussed.
\end{abstract}

DOI: 10.1103/PhysRevE.91.033016

PACS number(s): 47.55.Kf, 05.70.Ln, 83.50.Lh, 68.08.-p

\section{INTRODUCTION}

Suspensions of solid particles of microscale or nanoscale in liquids are ubiquitous in both nature and industries. The presence of solid particles, especially anisotropic ones, in a viscous fluid can result in fascinating rheological properties of the suspension [1-4]. Studies concerning the motion of an ellipsoidal particle in a viscous flow were initiated by Jeffery [5], who applied the no-slip boundary condition at particle surface and studied the particle revolution driven by a simple shear flow in the Stokes regime. The ellipsoidal particle was found to undergo a periodic revolution called the Jeffery orbit, which is controlled by the anisotropy (aspect ratio) of the particle. The analytical solution for the Jeffery orbit was derived by Jeffery himself and soon experimentally verified by Taylor [6]. Bretherton [7] demonstrated the general validity of the Jeffery orbit for particles of rotational symmetry, e.g., cylinders and fibers, followed by numerous experimental observations [8-10] and numerical simulations [11]. In addition, there have been investigations on the Jeffery orbit that involve wall effect [12-14], inertial effect [15-19], Poiseuille flow [20-22], axisymmetric random flow [23], and the coupling with heat flux [24]. Moreover, even under conditions that are not as ideal as in the original study, the Jeffery orbit dynamics can still be used to describe how the particle orientation responds to the fluid velocity gradient, covering the dynamics of nematic liquid crystals [25-30], and elastic particles [31-33], vesicles [34-36], and active particles [37] and nonflagellated $E$. coli in shear flows [38]. Recently, the study of microswimmer aggregation in various flows has attracted much attention

*Corresponding author: maqian@ust.hk because it is a fundamental element in microbial ecology [39], pathophoresis [40], and biofilm formation [41], and so on. For elongated microswimmers, their flow-driven motion always involves the Jeffery orbit [42]. Recent studies have revealed how their aggregations are affected by different flow types and their motilities in response to possible external stimuli [43-46], i.e., gyrotaxis, chemotaxis, phototaxis, and so on. All these studies demonstrate that the Jeffery orbit dynamics is a fundamental aspect of the motion of anisotropic objects in viscous flows. The Jeffery orbit was also used in the study of the viscosity of the ellipsoidal particle suspension, which was simplified to be dilute and free of Brownian motion and hydrodynamic interactions [5].

It is important to note that at the length scale of the dispersed particles (typically micrometer or nanometer), viscous force is typically dominant in comparison with inertial force, as characterized by the small Reynolds number $[47,48]$. The dominance of viscous force means that the hydrodynamic interactions are transformed almost instantaneously, resulting in certain constraints upon the motion of the concerned objects $[1,49,50]$. One of the benefits is that for a rigid-body motion in a viscous flow, some results can be obtained without directly solving it but by invoking the known results of some other motion [51]. These are the so-called hydrodynamic reciprocal relations $[1,49,52]$, also known as the Lorentz reciprocal theorem [53]. These relations can be regarded as a specific form of Onsager's reciprocal relations [54,55] in hydrodynamics and have been fruitfully employed in various studies, such as particle hydrodynamic interactions [56], propulsion of microorganisms by surface distortions [57], thermocapillary migration [58], Marangoni propulsion [59], electrophoresis [60], and flow over patterned surfaces [61].

In fluid dynamics, the Navier-Stokes equation has been constantly used together with the no-slip boundary condition. 
Though widely applied at the fluid-solid interface [47], the latter is actually a limit or a good approximation valid at macroscopic level and cannot be derived from fundamental principles [62,63]. Deviation from the no-slip condition has been reported in many studies, such as gas flows [64], non-Newtonian flows [63], moving contact lines [65], flows at micro- or nanoscale [66,67], and flows at high shear rate [68]. The Navier slip boundary condition was proposed to account for a tangential velocity of the fluid relative to the solid at the fluid-solid interface [69]. Recently, it has been shown that this slip condition can be derived from the principle of least energy dissipation [70]. Therefore, when boundary slip is taken into consideration, those celebrated classical results, obtained with the use of no-slip condition, have to be modified. To the best of our knowledge, there has been no report on the study of the Jeffery orbit with boundary slip though an interesting discussion was put forward [71]. As the Stokes equation and the Navier slip condition can both be derived from the principle of least energy dissipation, we expect that the hydrodynamic reciprocal relations can be preserved when the Navier slip condition is introduced to replace the no-slip condition. This understanding underpins the present work.

As to the numerical simulations for hydrodynamics at small scales, the Navier-Stokes equation is still valid as a continuum description [47], based on which numerous numerical methods have been developed for colloidal simulations [72-75]. As a powerful method for colloidal simulations, the fluid particle dynamics (FPD) method has been proposed by Tanaka and Araki [76,77]. This method employs a diffuse fluid-solid interface in order to avoid the explicit implementation of fluidparticle boundary condition. Instead, it approximates a solid particle as a highly viscous fluid such that the Navier-Stokes equation is solved in the whole space and the large particle number is no longer problematic. In the present work, we apply the Navier slip condition at particle surface. This is to be realized by inserting a thin layer of fluid with reduced viscosity between the solid particle and the surrounding liquid. This treatment can be readily incorporated into the FPD method, resulting in an effective slip length, which measures the extent of boundary slip [78,79].

The purpose of the present work is to investigate the effects of boundary slip on the orientational motion of an anisotropic particle in a simple shear flow. A fluid-particle system is studied where an anisotropic particle is immersed in a viscous fluid confined between two shearing walls. We first generalize the hydrodynamic reciprocal relations to replace the no-slip condition with the Navier slip condition. We then apply these relations to the fluid-particle system with a slippery elliptical particle. The coefficient for the cross coupling between the rotational torque and the shear stress is identified and related to the particle's effective anisotropy. The Jeffery orbit of this particle is also studied, in which the same coefficient appears. We carry out numerical simulations using the FPD method. The dependence of the cross-coupling coefficient on the slip length at particle surface is measured, showing that the boundary slip can enhance the effective anisotropy of the particle and hence the cross coupling between the rotational torque and the shear stress. We further consider the Jeffery orbit of a circular patchy particle which possesses the symmetry of an ellipse via surface patterning of no-slip and slip patches.
Numerical results show that its effective anisotropy depends on the pattern geometry and the slip length. Finally, there is a brief discussion on the connection of the present work to nematic liquid crystals' constitutive relations.

The paper is organized as follows. In Sec. II, Onsager's variational principle and reciprocal relations are reviewed. The Stokes equation and the Navier slip boundary condition are shown to be derivable from Onsager's principle. Then the hydrodynamic reciprocal relations are generalized by replacing the no-slip condition with the Navier slip condition. In Sec. III, the FPD method is presented, with extension for applying the Navier slip condition. In Sec. IV, the fluidparticle system with a slippery elliptical particle is investigated for the hydrodynamic reciprocal relations and Jeffery orbit. The Jeffery orbit of a circular patchy particle is studied as well. In Sec. V, the fluid-particle system is shown to be analogous to a unit of nematic liquid crystal regarding the cross coupling between the rotational torque and the shear stress. The paper is concluded in Sec. VI with a few remarks. The numerical implementation of the FPD method is outlined in the Appendix.

\section{ONSAGER'S VARIATIONAL PRINCIPLE AND HYDRODYNAMIC RECIPROCAL RELATIONS}

\section{A. Onsager's variational principle and reciprocal symmetry}

Consider a closed system not far from equilibrium. The fluctuations of a set of coarse-grained variables $\alpha \equiv\left\{\alpha_{i}\right\}$ are measured relative to their most probable (equilibrium) values $\left\{\alpha_{i}=0\right\}$. The entropy of the system $S$, which reaches its maximum $S_{e}$ at equilibrium, can be expressed in the quadratic form

$$
S=S_{e}+\Delta S(\alpha)=S_{e}-\frac{1}{2} \beta_{i j} \alpha_{i} \alpha_{j},
$$

where $\beta_{i j}$ form a symmetric and positive definite matrix. Here the Einstein summation convention is used. The probability density at state $\alpha$ is related to $\Delta S(\alpha)$ via $f(\alpha)=$ $f_{0} \exp \left[\Delta S(\alpha) / k_{B}\right]$, with $k_{B}$ being the Boltzmann constant. When the system deviates from equilibrium, spontaneous irreversible processes arise in response to the thermodynamic force $X_{i}$ conjugate to $\alpha_{i}$ :

$$
X_{i} \equiv \frac{\partial \Delta S}{\partial \alpha_{i}}=-\beta_{i j} \alpha_{j},
$$

which is linear in $\alpha$ due to the quadratic form of $\Delta S(\alpha)$ in Eq. (1).

For small deviation from equilibrium, the system is in the linear response regime, where the state $\alpha$ evolves according to the kinetic equations

$$
\dot{\alpha}_{i}=L_{i j} X_{j}
$$

or, equivalently,

$$
X_{i}=R_{i j} \dot{\alpha}_{j}
$$

where the kinetic coefficients $L_{i j}$ form a symmetric and positive definite matrix and so do the coefficients $R_{i j}$, with $L_{i j} R_{j k}=\delta_{i k}$. Off-diagonal entries $L_{i j}$ and $R_{i j}$ are referred to as cross-coupling coefficients between different irreversible processes labeled by $i$ and $j$. Under the condition that the variables $\alpha$ are even, i.e., their signs remain invariant 
under time-reversal operation, Onsager derived the reciprocal relations

$$
L_{i j}=L_{j i}
$$

and, consequently, $R_{i j}=R_{j i}$, from the microscopic reversibility $[54,55]$. It is worth emphasizing that his derivation does not require detailed knowledge of the irreversible processes. Some experimental verifications have been reported by Miller [80].

Based on Onsager's reciprocal relations (5), the kinetic equations (4) can be used to formulate a variational principle governing the irreversible processes. This variational principle states that, for a closed system, the state evolution equations can be obtained by maximizing the action function (or functional),

$$
\dot{S}(\alpha, \dot{\alpha})-\Phi_{S}(\dot{\alpha}, \dot{\alpha})
$$

with respect to the rates $\left\{\dot{\alpha}_{i}\right\}$. Here $\dot{S}=X_{i} \dot{\alpha}_{i}$ is the rate of change of the entropy, and the dissipation function $\Phi_{S}(\dot{\alpha}, \dot{\alpha})=$ $R_{i j} \dot{\alpha}_{i} \dot{\alpha}_{j} / 2$ is half the rate of entropy production. For an open system, however, there is an additional term $\dot{S}^{*}$, which is the rate of entropy given by the system to the environment, added to the action (6), leading to

$$
\mathcal{O}=\dot{S}+\dot{S}^{*}-\Phi_{S}(\dot{\alpha}, \dot{\alpha}),
$$

which is called the Onsager-Machlup action [54,55,81]. Note that $\dot{S}+\dot{S}^{*}$ is still linear in $\left\{\dot{\alpha}_{i}\right\}$. Onsager's variational principle states that for an open system, the state evolution equations can be obtained by maximizing the Onsager-Machlup action $\mathcal{O}$ with respect to the rates $\left\{\dot{\alpha}_{i}\right\}$. This principle serves as a general framework for describing irreversible processes in the linear response regime.

Onsager's variational principle is an extension of Rayleigh's principle of least energy dissipation [82], and, naturally, it reduces to the latter for isothermal systems. In an isothermal system, the rate of entropy given by the system to the environment can be expressed as $\dot{S}^{*}=-\dot{Q} / T=-\dot{U} / T$, where $T$ is the system temperature, $\dot{Q}$ is the rate of heat transfer from the environment to the system, and $\dot{U}$ is the rate of change of the system energy, with $\dot{Q}=\dot{U}$ according to the first law of thermodynamics. Note that $T$ is constant here. The maximization of the Onsager-Machlup action (7) is equivalent to the minimization of the so-called Rayleighian,

$$
\mathcal{R}=\dot{F}(\alpha, \dot{\alpha})+\Phi_{F}(\dot{\alpha}, \dot{\alpha}),
$$

with respect to the rates $\left\{\dot{\alpha}_{i}\right\}$. Here $\dot{F} \equiv \dot{U}-T \dot{S}=-T(\dot{S}+$ $\left.\dot{S}^{*}\right)$ is the rate of change of the Helmholtz free energy of the system, and the dissipation function $\Phi_{F}(\dot{\alpha}, \dot{\alpha}) \equiv T \Phi_{S}(\dot{\alpha}, \dot{\alpha})$ is half the rate of free-energy dissipation. As $\dot{F}$ is linear while $\Phi_{F}$ is quadratic in the rates $\left\{\dot{\alpha}_{i}\right\}$, the principle of least energy dissipation leads to $\dot{F}=-2 \Phi_{F}$. For isothermal systems, the Rayleighian can be written as

$$
\mathcal{R}=\frac{\partial F}{\partial \alpha_{i}} \dot{\alpha}_{i}+\frac{1}{2} \zeta_{i j} \dot{\alpha}_{i} \dot{\alpha}_{j},
$$

where the first term in the right-hand side is $\dot{F}$ and the second term is $\Phi_{F}(\dot{\alpha}, \dot{\alpha})$, which is in a quadratic form with the friction coefficients $\zeta_{i j}$ forming a symmetric and positive-definite matrix. Minimization of $\mathcal{R}$ with respect to the rates gives the kinetic equations

$$
-\frac{\partial F}{\partial \alpha_{i}}=\zeta_{i j} \dot{\alpha}_{j},
$$

which can be interpreted as a balance between the reversible force $-\partial F / \partial \alpha_{i}$ and the dissipative force linear in the rates.

It is worth emphasizing that although the variational principle is equivalent to the kinetic equations combined with the reciprocal relations, the former possesses a notable advantage [83]: The variational form allows flexibility in the choice of state variables. Once these variables are chosen, the conjugate forces are generated automatically via calculus of variations.

\section{B. Stokes equation and Navier slip boundary condition from the variational principle}

The variational principle is now fully employed to investigate the Stokes flows with boundary slip. Below is a brief review, showing that the Stokes equation and the Navier slip boundary condition can be derived from the principle of least energy dissipation [70].

Consider an incompressible Newtonian fluid in a region $\Omega$ with a solid boundary $\partial \Omega$, and neglect the inertial effect. The incompressibility condition reads $\vec{\nabla} \cdot \vec{v}=0$, and the boundary is impermeable at which the normal velocity $\left.v_{n}\right|_{\partial \Omega}=0$. Here the rate is the velocity field $\vec{v}(\vec{r})$ and the free energy is constant in time. The dissipation in the bulk region is due to the viscosity $\eta$, and the corresponding dissipation function is [47]

$$
\Phi_{v}=\int_{\Omega} d V \frac{1}{4} \eta\left(\partial_{i} v_{j}+\partial_{j} v_{i}\right)^{2} .
$$

If boundary slip occurs at the fluid-solid interface, then the corresponding dissipation function is given by

$$
\Phi_{s}=\int_{\partial \Omega} d S \frac{1}{2} \beta\left(\vec{v}^{\text {slip }}\right)^{2},
$$

where $\beta$ is the slip coefficient, and $\vec{v}^{\text {slip }}$ is the slip velocity, defined as the tangential velocity of the fluid relative to the solid at the fluid-solid interface. Here the solid boundary is still and hence $\vec{v}^{\text {slip }}=\vec{v}$. The Rayleighian of the system is given by

$$
\begin{aligned}
\mathcal{R} & =\Phi_{v}+\Phi_{s} \\
& =\int_{\Omega} d V \frac{1}{4} \eta\left(\partial_{i} v_{j}+\partial_{j} v_{i}\right)^{2}+\int_{\partial \Omega} d S \frac{1}{2} \beta \vec{v}^{2} .
\end{aligned}
$$

Combining the principle of least energy dissipation with the incompressibility condition, we have $\delta\left[\mathcal{R}-\int_{\Omega} d V \pi \partial_{i} v_{i}\right]=0$ for any $\vec{v}(\vec{r}) \rightarrow \vec{v}(\vec{r})+\delta \vec{v}(\vec{r})$, with $\pi$ being the Lagrange multiplier. The Euler-Lagrange equations are the Stokes equation

$$
-\vec{\nabla} \pi+\vec{\nabla} \cdot\left[\eta\left(\vec{\nabla} \vec{v}+\vec{\nabla} \vec{v}^{T}\right)\right]=0
$$

in the bulk region and the Navier boundary condition

$$
\hat{n} \cdot \stackrel{\leftrightarrow}{\sigma} \text { vis } \cdot \stackrel{\leftrightarrow}{\tau}+\beta \vec{v}^{\text {slip }}=0
$$

at the solid boundary, where $\stackrel{\leftrightarrow}{\sigma}$ vis $\equiv \eta\left(\vec{\nabla} \vec{v}+\vec{\nabla} \vec{v}^{T}\right)$ is the viscous stress tensor and $\overleftrightarrow{\tau} \equiv \overleftrightarrow{I}-\hat{n} \hat{n}$ with $\hat{n}$ being the outward pointing (from fluid into solid) unit vector normal 
to $\partial \Omega$. Note that the Lagrange multiplier $\pi$ is the pressure. The total stress is $\overleftrightarrow{\sigma} \equiv-\pi \overleftrightarrow{I}+\overleftrightarrow{\sigma}^{\text {vis }}$.

\section{Lorentz reciprocal theorem}

Prior to Onsager's general work, there existed a few specific reciprocal relations studied by Lord Kelvin and Helmholtz. In fluid dynamics, the hydrodynamic reciprocal relations, known as the Lorentz reciprocal theorem [49], are also regarded as a special form of Onsager's reciprocal relations [84]. Consider an incompressible Stokes flow in a region $\Omega$ with a solid boundary $\partial \Omega$. The velocity field $\vec{v}(\vec{r})$ is governed by the Stokes equation (14), with the no-slip boundary condition at $\partial \Omega$. Suppose that in the same system, two velocity fields $\vec{v}^{(1)}$ and $\vec{v}^{(2)}$ are both the solutions to Eq. (14), with their corresponding stress fields denoted by $\stackrel{\leftrightarrow}{\sigma}^{(1)}$ and $\overleftrightarrow{\sigma}^{(2)}$, respectively. The Lorentz reciprocal theorem states that

$$
\int_{\partial \Omega} d S \hat{n} \cdot \overleftrightarrow{\sigma}^{(1)} \cdot \vec{v}^{(2)}=\int_{\partial \Omega} d S \hat{n} \cdot \stackrel{\leftrightarrow}{\sigma}^{(2)} \cdot \vec{v}^{(1)},
$$

where $\hat{n}$ is the outward pointing (from fluid into solid) unit vector normal to $\partial \Omega$. The proof is as follows. The left-hand side of Eq. (16) can be expressed as

$$
\begin{aligned}
& \int_{\partial \Omega} d S \hat{n} \cdot \overleftrightarrow{\sigma}^{(1)} \cdot \vec{v}^{(2)} \\
& =\int_{\Omega} d V \vec{\nabla} \cdot\left[\overleftrightarrow{\sigma}^{(1)} \cdot \vec{v}^{(2)}\right] \\
& =\int_{\Omega} d V\left[\vec{\nabla} \cdot \overleftrightarrow{\sigma}^{(1)} \cdot \vec{v}^{(2)}+\overleftrightarrow{\sigma}^{(1)}: \vec{\nabla} \vec{v}^{(2)}\right] \\
& =\int_{\Omega} d V\left\{-p^{(1)} \delta_{i j} \partial_{i} v_{j}^{(2)}+\frac{\eta}{2}\left[\partial_{i} v_{j}^{(1)}+\partial_{j} v_{i}^{(1)}\right]\right. \\
& \left.\times\left[\partial_{i} v_{j}^{(2)}+\partial_{j} v_{i}^{(2)}\right]\right\} \\
& =\int_{\Omega} d V \frac{\eta}{2}\left[\partial_{i} v_{j}^{(1)}+\partial_{j} v_{i}^{(1)}\right]\left[\partial_{i} v_{j}^{(2)}+\partial_{j} v_{i}^{(2)}\right] \text {, }
\end{aligned}
$$

where the Stokes equation, $\vec{\nabla} \cdot \vec{v}=0$, and the symmetry of $\stackrel{\leftrightarrow}{\sigma}$ are used. It is readily seen that the right-hand side of of Eq. (16) leads to the same expression. Furthermore, the Lorentz reciprocal theorem (16) can be expressed as

$$
F_{k}^{(1)} \dot{x}_{k}^{(2)}=F_{k}^{(2)} \dot{x}_{k}^{(1)},
$$

where $\dot{x}_{k}$ are the generalized velocities of the solid objects and $F_{k}$ are the generalized dissipative forces conjugate to $\dot{x}_{k}$ [85]. Note that the no-slip boundary condition is applied to move from $\vec{v}$ of the fluid to $\dot{x}_{k}$ of the solid. Due to the linearity of the Stokes flows, we have the linear dependence of the forces on the rates:

$$
F_{k}=\zeta_{k l} \dot{x}_{l}
$$

where the friction coefficients $\zeta_{k l}$ form a positive definite matrix. It follows from Eq. (18) that the Lorentz reciprocal theorem can be expressed as

$$
\zeta_{k l}=\zeta_{l k},
$$

meaning that the matrix formed by the friction coefficients $\zeta_{k l}$ is symmetric.
In the above discussion, the Lorentz reciprocal theorem expressed in Eqs. (18) and (20) is derived from the Stokes equation and the no-slip boundary condition. We have already shown that the Stokes equation (14) and the Navier boundary condition (15) can be simultaneously obtained from the principle of least energy dissipation. It is therefore expected that the hydrodynamic reciprocal relations can be generalized to describe the Stokes flows with the Navier boundary condition.

Consider the same system with the Navier boundary condition. The velocity of the solid boundary $\partial \Omega$ is denoted by $\vec{W}$. From the Navier boundary condition (15), we readily obtain

$$
\int_{\partial \Omega} d S \hat{n} \cdot \overleftrightarrow{\sigma}^{(1)} \cdot \vec{v}^{\operatorname{slip}(2)}=\int_{\partial \Omega} d S \hat{n} \cdot \overleftrightarrow{\sigma}^{(2)} \cdot \vec{v}^{\operatorname{slip}(1)}
$$

Meanwhile, Eq. (16) still holds. Note that $\vec{W}=\vec{v}-\vec{v}^{\text {slip }}$ on $\partial \Omega$. By combining Eqs. (16) and (21), we obtain the generalized form of the hydrodynamic reciprocal relations,

$$
\int_{\partial \Omega} d S \hat{n} \cdot \overleftrightarrow{\sigma}^{(1)} \cdot \vec{W}^{(2)}=\int_{\partial \Omega} d S \hat{n} \cdot \overleftrightarrow{\sigma}^{(2)} \cdot \vec{W}^{(1)}
$$

Note that the no-slip limit is obtained as $\beta \rightarrow \infty$ with $\vec{W}=\vec{v}$ on $\partial \Omega$. With the Lorentz reciprocal theorem generalized from Eq. (16) to (22), it can be further expressed as Eq. (18), which results in the symmetry in Eq. (20). We emphasize that in the presence of boundary slip, we need Eq. (22) in order to arrive at Eqs. (18) and (20). It is remarkable that the reciprocal symmetry is preserved in the Stokes flows with the Navier slip condition.

To use Eq. (20) for the present study, we consider the solid boundary $\partial \Omega$ consisting of the surfaces of $N$ rigid bodies $\partial \Omega^{i}$ $(i=1, \ldots, N)$, each in a motion described by the translational velocity $\vec{V}^{i}$ and the angular velocity $\vec{\omega}^{i}$. The solid velocity at $\vec{r}$ on $\partial \Omega^{i}$ can be expressed as

$$
\vec{W}(\vec{r})=\vec{V}^{i}+\vec{\omega}^{i} \times \delta \vec{r}^{i},
$$

where $\delta \vec{r}^{i}$ is measured relative to the center of mass of the $i$-th rigid body. Then we have

$$
\begin{aligned}
\int_{\partial \Omega} d S \hat{n} \cdot \overleftrightarrow{\sigma}^{(1)} \cdot \vec{W}^{(2)} & \sum_{i=1}^{N}\left[\int_{\partial \Omega^{i}} d S \hat{n} \cdot \overleftrightarrow{\sigma}^{(1)}\right] \cdot \vec{V}^{i(2)} \\
= & +\sum_{i=1}^{N}\left\{\int_{\partial \Omega^{i}} d S \delta \vec{r}^{i} \times\left[\hat{n} \cdot \overleftrightarrow{\sigma}^{(1)}\right]\right\} \cdot \vec{\omega}^{i(2)}
\end{aligned}
$$

where $\int_{\partial \Omega^{i}} d S \hat{n} \cdot \stackrel{\leftrightarrow}{\sigma}$ is the total force by the $i$-th rigid body on the fluid and $\int_{\partial \Omega^{i}} d S \delta \vec{r}^{i} \times(\hat{n} \cdot \stackrel{\leftrightarrow}{\sigma})$ is the total torque by the $i$-th rigid body on the fluid. This leads to a specific form of Eq. (18), in which $\vec{V}^{i}$ and $\vec{\omega}^{i}(i=1, \ldots, N)$ are the generalized velocities of the rigid bodies $\dot{x}_{k}$ and $\int_{\partial \Omega^{i}} d S \hat{n} \cdot \overleftrightarrow{\sigma}$ and $\int_{\partial \Omega^{i}} d S \delta \vec{r}^{i} \times(\hat{n} \cdot \overleftrightarrow{\sigma})$ are their conjugate generalized forces $F_{k}$.

We point out that the Lorentz reciprocal theorem is valid only when the slip length $l_{s}=\eta / \beta$ is a material constant, which makes the Navier boundary condition linear [86]. In 
fact, nonlinear slip boundary conditions have been reported in the literature [68,87-89], where the slip length $l_{s}$ depends on the shear rate at the solid boundary $\dot{\gamma}$ when the latter is large enough. For example, the rate-dependent slip length $l_{s}=l_{s}^{0}\left(1-\dot{\gamma} / \dot{\gamma}_{c}\right)^{-1 / 2}$ has been reported [87], where $l_{s}^{0}$ is the constant slip length at low $\dot{\gamma}$, and $\dot{\gamma}_{c}$ is the critical shear rate at which $l_{s}$ diverges. For $\dot{\gamma} \ll \dot{\gamma}_{c}, l_{s}$ is almost a constant $\left(\simeq l_{s}^{0}\right)$, and the Navier boundary condition is recovered. However, when the nonlinear effect occurs at high $\dot{\gamma}$, Eq. (21) no longer holds, and, consequently, the Lorentz reciprocal theorem expressed in Eq. (22), (18), or (20) breaks down.

\section{NUMERICAL METHOD}

The FPD method is employed in our numerical simulations. Proposed by Tanaka and Araki [76] for colloidal suspension simulations, this method has been further developed to treat numerous problems of liquid-solid mixtures [77]. It has been shown to be an efficient and powerful method for simulations of solid particle dynamics in fluids because of the accurate inclusion of hydrodynamic interactions, the absence of explicit implementation of the boundary conditions at fluid-solid interface, and the insensitivity of the computational complexity to the particle number. In this section, there is a brief review of the FPD method followed by an extension to include the boundary slip at fluid-solid interface.

\section{A. Fluid particle dynamics method}

The FPD method can be regarded as a hybrid method. Two sets of variables are employed: (i) the on-lattice variables including the velocity field $\vec{v}(\vec{r})$, the pressure field $p(\vec{r})$, the composition field $\phi(\vec{r})$, and the viscosity field $\eta(\vec{r})$ and (ii) the off-lattice variables including the particle's center-ofmass position $\vec{R}_{p}$, the particle's orientation $\vec{d}_{p}$, the particle's center-of-mass velocity $\vec{V}_{p}$, and the particle's angular velocity $\vec{\omega}_{p}$. On the one hand, a particle is represented by $\vec{R}_{p}$ and $\vec{d}_{p}$, and, on the other hand, it is also represented by the composition field given by

$$
\phi(\vec{r})=\frac{1}{2}\left\{\tanh \left[\frac{d(\vec{r})}{\xi}\right]+1\right\},
$$

where $\xi$ is a small length scale characterizing the fluid-solid interfacial thickness and $d(\vec{r})$ is the signed distance to the fluidsolid interface, which is positive inside and negative outside the solid particle. This results in $\phi=1$ in the solid region and $\phi=$ 0 in the fluid region, with the fluid-solid interface defined as the locus of $\phi=0.5$. In colloidal suspension simulations, the longrange and many-body hydrodynamic interactions are of critical importance. The FPD method treats the solid particles as a highly viscous incompressible fluid in which the velocity field becomes very close to that of a rigid body $[76,77]$. Momentum transfer between the fluid and the solid particles is realized by solving the Navier-Stokes equation,

$$
\rho \frac{D \vec{v}}{D t}=-\vec{\nabla} \pi+\vec{\nabla} \cdot\left[\eta\left(\vec{\nabla} \vec{v}+\vec{\nabla} \vec{v}^{T}\right)\right]+\vec{f},
$$

where the fluid and solid are assumed to be of the same density $\rho$, the viscosity $\eta$ depends on $\phi$ with $\eta(\phi)=\eta_{s}$ as the "solid" viscosity for $\phi=1$ and $\eta(\phi)=\eta_{l}$ as the liquid viscosity for $\phi=0$, and $\vec{f}$ is the external force density. From the velocity field in the particle region, the average velocity and vorticity are measured to give $\vec{V}_{p}$ and $\vec{\omega}_{p}$, respectively. The implementation details can be found in the Appendix. Tanaka and Araki used $\eta(\phi)$ as a linear function of $\phi$ to achieve the no-slip condition at fluid-solid interface [77].

There are two dimensionless numbers crucial to the validity of the FPD method: the viscosity ratio $r_{\eta} \equiv \eta_{s} / \eta_{l}$ and the relative interfacial thickness $r_{\xi} \equiv \xi / a$, with $a$ being a characteristic length for the particle. In general, better simulation results are obtained for larger $r_{\eta}$ and smaller $r_{\xi}$ [76,77]. In the numerical simulations reported here, $r_{\eta}=50$ and $r_{\xi}<0.1$ are used.

In our simulations, the particle may be expected to undergo a motion with prescribed translational velocity $\vec{V}^{*}$ and angular velocity $\vec{\omega}^{*}$. This is to be realized by employing a simple interpolation approach. The rigid-body velocity distribution in the particle region reads

$$
\vec{v}_{r}(\vec{r})=\vec{V}^{*}+\vec{\omega}^{*} \times\left(\vec{r}-\vec{R}_{p}\right) .
$$

To update the velocity distribution from $\vec{v}^{n}$ to $\vec{v}^{n+1}$, we let $\vec{v}^{n+1}$ be an interpolation $[90,91]$ between $\vec{v}_{r}$ and the intermediate velocity field $\vec{v}_{i}^{n}$ which is solved from the Navier-Stokes equation (25) with $\vec{v}^{n}$ as the initial condition, i.e.,

$$
\vec{v}^{n+1}(\vec{r})=\Theta[\phi(\vec{r})] \vec{v}_{r}(\vec{r})+\{1-\Theta[\phi(\vec{r})]\} \vec{v}_{\mathrm{i}}^{n}(\vec{r}),
$$

in which $\Theta(\phi)$ is a Heaviside function with $\Theta=1$ for $\phi \geqslant \phi_{c}$ and 0 otherwise. With the understanding that $\phi_{c}$ should be close to $\phi_{s}=1, \phi_{c}=0.95$ is used in our simulations. Numerical tests show that for $\phi_{c}$ values close to 1, numerical results converge.

\section{B. Boundary slip at fluid-solid interface}

It has been shown that using a monotonic function $\eta(\phi)$ across the fluid-solid interfacial region will reproduce the no-slip boundary condition [77]. However, at the microor nanoscale, boundary slip occurs in many circumstances. Here we introduce a slippery fluid-solid interface to the FPD method. It has been demonstrated that a thin layer of fluid ("lubricant") with lower viscosity between the fluid and the solid can effectively generate a slip across the fluid-solid interface $[78,79]$. Consider a thin layer of lubricant with a very small viscosity $\eta_{i}$ and a width comparable to the interfacial thickness $\xi$. Let $\eta_{l}$ denote the fluid viscosity and $l_{s}$ denote the slip length in the Navier boundary condition which is to be realized across the fluid-solid interface. The continuity of the shear stress across the interface means $\eta_{l} \dot{\gamma} \sim \eta_{i} \Delta v / \xi$, where $\dot{\gamma}$ is the shear rate at the solid boundary and $\Delta v$ is the change of tangential velocity across the interfacial region. With the understanding that $\Delta v$ acts as the slip velocity, we have the slip coefficient $\beta \sim \eta_{i} / \xi$, which gives the slip length $l_{s}=\eta_{l} / \beta \sim\left(\eta_{l} / \eta_{i}\right) \xi$. This leads to the scaling relation [79]

$$
\frac{l_{s}}{\xi} \sim \frac{\eta_{l}}{\eta_{i}},
$$

which shows that a small interfacial viscosity $\eta_{i}$ (being much smaller than $\eta_{l}$ ) can result in a large slip length $(\gg \xi)$. 
In our simulations, we use

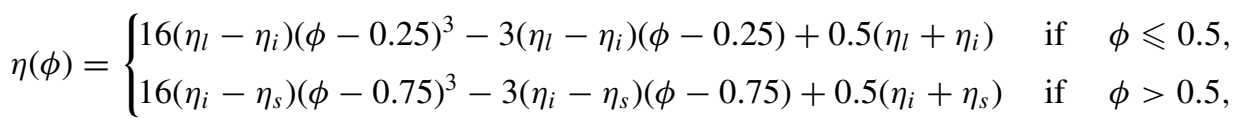

with $\eta(0)=\eta_{l}, \eta(0.5)=\eta_{i}$, and $\eta(1)=\eta_{s}$ for the thin layer of lubricant between the fluid and the solid. Expected to follow the scaling relation (28), the quantitative relation between $l_{s}$ and $\eta_{i}$ has to be numerically measured. For this purpose, we generate a tangential flow of the fluid over the solid surface, with the lubricant layer in the interfacial region. The velocity profile is measured, from which the slip length $l_{s}$ is obtained through an extrapolation. By varying $\eta_{i}$, we can obtain $l_{s}$ as a function of $\eta_{i}$, which is shown in Fig. 1. For $l_{s} \geqslant 3 \xi$, a linear fitting gives

$$
\frac{l_{s}}{\xi}=0.412+0.783 \frac{\eta_{l}}{\eta_{i}},
$$

which agrees with the relation (28) well. For $l_{s} \simeq \xi$, however, the scaling relation (28) no longer holds. In fact, due to the diffuse nature of the interface, the smallest slip length that can be accurately determined should be of the order of magnitude of $\xi$. Finally, it is worth pointing out that a specific form of interpolation for $\eta(\phi)$ results in a specific realization of slip: the parameters $s_{0}$ and $s_{1}$ in $l_{s} / \xi=s_{0}+s_{1} \eta_{l} / \eta_{i}$ may be varied by changing the viscosity function $\eta(\phi)$. But the scaling relation (28) is always satisfied.

In the next section, we use the viscosity function (29) to introduce slip at particle surface. We also use Eq. (30) to directly obtain $l_{s}$ from $\eta_{i}$. Since this equation is numerically obtained for flat solid surfaces, it is therefore expected to be valid when the curvature radius of the particle surface is much larger than the interfacial thickness $\xi$.

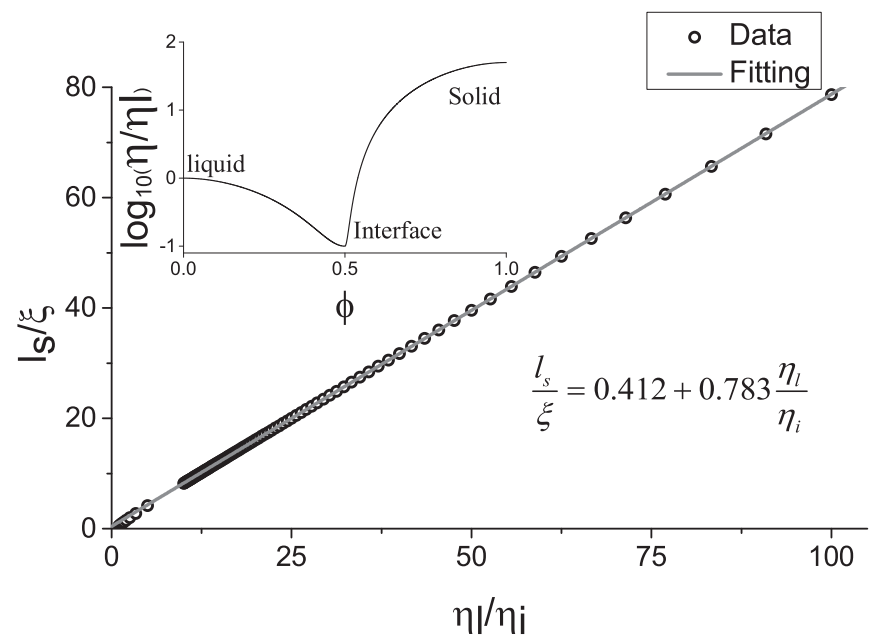

FIG. $1 . l_{\mathrm{s}} / \xi$ plotted as a function of $\eta_{l} / \eta_{i}$. The symbols represent the results from the FPD simulations with slippery fluid-solid interface; the solid line is a linear fitting expressed by Eq. (30) for $l_{s} / \xi \geqslant 3$. The inset shows $\log _{10}\left(\eta / \eta_{l}\right)$ plotted as a function of $\phi$ for $\eta(\phi)$ in Eq. (29), with $\eta_{s} / \eta_{l}=50$ and $\eta_{i} / \eta_{l}=0.1$.

\section{NUMERICAL SIMULATIONS AND RESULTS}

\section{A. Hydrodynamic reciprocal relations in a fluid-particle system}

Consider a two-dimensional fluid-particle system as shown in Fig. 2. An elliptical particle is immersed in a viscous fluid confined between two solid walls parallel to the $x$ axis. The particle is positioned at the center between the two walls. The two walls shear the viscous fluid with the speed $W$ in the $\pm x$ directions, and the particle rotates with an angular velocity $\omega \hat{z}$ in the $x y$ plane. Here $\hat{z}$ is the unit vector in the $z$ direction. We use $\hat{n}$ to denote the outward pointing (from fluid into solid) unit vector normal to the particle surface, $a$ to denote the major axis, and $b$ to denote the minor axis of the ellipse, with the aspect ratio of the particle defined by $e \equiv b / a<1$.

Physically, the integrated stress $S \equiv \int_{\mathrm{uw}} \sigma_{y x} d x$ is the tangential force by the upper wall on the fluid (with "uw" in $\int_{\text {uw }}$ denoting the upper wall), and $T \equiv \int_{p} \hat{z} \cdot[\vec{r} \times(\hat{n} \cdot \stackrel{\leftrightarrow}{\sigma})] d S$ is the torque by the solid particle on the fluid (with " $\mathrm{p}$ " in $\int_{p}$ denoting the particle). Considering the top-bottom symmetry, we take the wall velocity variation $2 W$ and the particle angular velocity $\omega$ as the two rates, with $S$ and $T$ as their conjugate forces. The rate of dissipation can be written as

$$
D=2 W S+\omega T \text {. }
$$

As the system is linear, we have the linear dependence of the forces on the rates:

$$
\left[\begin{array}{l}
S \\
T
\end{array}\right]=\left[\begin{array}{cc}
\alpha & \gamma_{S} \\
\gamma_{T} & \beta
\end{array}\right]\left[\begin{array}{c}
2 W \\
\omega
\end{array}\right]
$$

where $\alpha, \beta, \gamma_{S}$, and $\gamma_{T}$ are the friction coefficients defined in Eq. (19). The corresponding hydrodynamic reciprocal

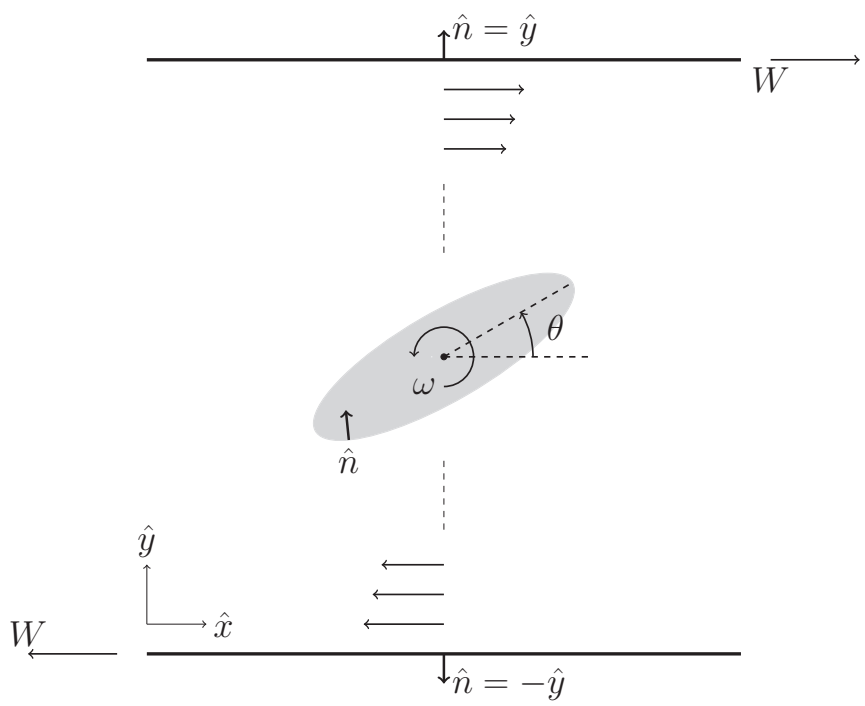

FIG. 2. A schematic illustration for a two-dimensional fluidparticle system. 
relation is

$$
\gamma_{S}=\gamma_{T}
$$

This symmetry has been proved in the previous section, and its numerical verification can actually be used as a test of validity for our simulations.

According to Eq. (32), $\gamma_{S}$ can be measured using the relation $\gamma_{S}=S_{W=0} / \omega$, in which $W=0$ represents the case where the two walls are fixed (with $W=0$ ) and the particle rotates (with an angular velocity $\omega$ ). This is termed case 1 . Similarly, $\gamma_{T}$ can be measured using the relation $\gamma_{T}=T_{\omega=0} /(2 W)$, in which $\omega=0$ represents the case where the particle is fixed (with $\omega=0)$ and the two walls move with the velocities $\pm W \hat{x}$. This is termed case 2 .

The cross-coupling coefficients $\gamma_{S}$ and $\gamma_{T}$ depend on the particle orientation $\theta$ (see Fig. 2). If the distance between the two walls $H$ is large compared to the particle dimensions, then the flow far away from the particle can be regarded as a simple shear flow with the shear rate given by $\dot{\gamma}=2 \mathrm{~W} / \mathrm{H}$. This simple shear flow can be decomposed into two parts [49]: an isotropic rotation field with angular velocity $-\dot{\gamma} / 2$ and an anisotropic distortion field without volumetric change. The latter consists of an elongation along $1 / \sqrt{2}(\hat{x}+\hat{y})$ direction and a compression along $1 / \sqrt{2}(-\hat{x}+\hat{y})$ direction, both of rate $\dot{\gamma} / 2$.

Positioned at the center, the particle undergoes no translational motion. The torque exerted by the particle on the fluid scales as

$$
T \propto\left(\omega+\frac{\dot{\gamma}}{2}\right)-\frac{\dot{\gamma}}{2} p \cos 2 \theta,
$$

in which $p$ is a dimensionless parameter measuring the anisotropy of the particle. This expression is obtained by considering $T$ as a sum of two distinct contributions [92]. The first contribution is from the angular velocity of the particle relative to the fluid, given by $\omega-(-\dot{\gamma} / 2)$. The second contribution is from the mismatch between the particle orientation and the principal axes of the distortion field, from which $\cos 2 \theta$ arises. Note that the first contribution $\omega+\dot{\gamma} / 2$ is isotropic, whereas the second contribution $-(\dot{\gamma} / 2) p \cos 2 \theta$ is anisotropic ( $\theta$ dependent) with the anisotropy measured by $p$. According to Jeffery [5], the dimensionless parameter $p$ is given by

$$
p=\frac{1-e^{2}}{1+e^{2}}
$$

for an elliptical particle with no-slip boundary condition, with $p \rightarrow 0$ for $e \rightarrow 1$ (circular particle) and $p \rightarrow 1$ for $e \rightarrow 0$ (slender beam). To the best of our knowledge, there has been no report of the effect of boundary slip on the dimensionless parameter $p$.

For $H \gg a$, Eq. (34) can be used to give the angular dependence of the cross-coupling coefficients

$$
\gamma_{S}=\gamma_{T} \equiv \frac{T_{\omega=0}}{2 W} \propto 1-p \cos 2 \theta,
$$

with $T_{\omega=0} \propto(1-p \cos 2 \theta) \dot{\gamma} / 2$ and $\dot{\gamma}=2 W / H$. It is worth emphasizing that this expression holds for particles with either no-slip or the Navier slip boundary condition. Below we numerically demonstrate the effect of boundary slip on
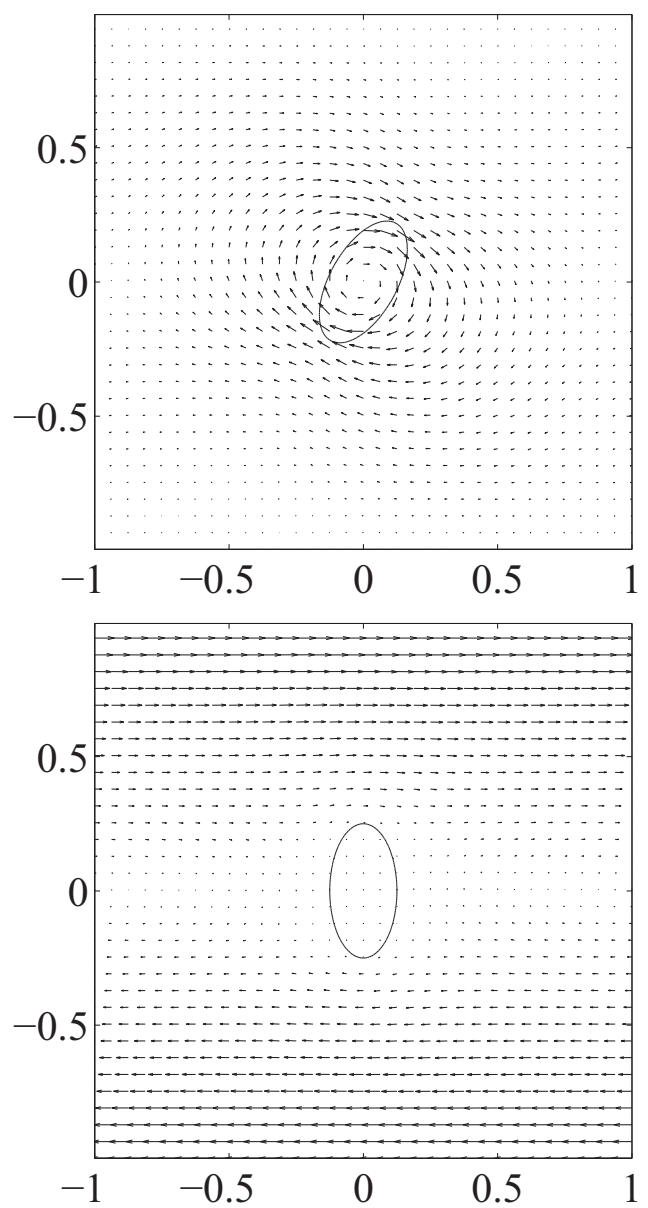

FIG. 3. Snapshots of the velocity field (arrows) and the particle (level curve of $\phi=0.5$ ), obtained for the numerical verification of the hydrodynamic reciprocal relations. (a) Case 1 where the two walls are fixed (with $W=0$ ) and the elliptical particle rotates (with angular velocity $\omega$ ). (b) Case 2 where the particle is fixed (with $\omega=0$ ) and the two walls move with the velocities $\pm W \hat{x}$. The slip length is $l_{s}=5 \xi$. The fluid-particle system measures $2 \times 2$, the major axis of the particle is $a=0.25$, and the aspect ratio is $e=0.5$. The fluid-solid interfacial thickness is $\xi=0.01$, the viscosity ratio is $r_{\eta} \equiv \eta_{s} / \eta_{l}=$ 50 , the angular velocity in case 1 is $\omega=-1$, and the wall speed in case 2 is $W=2$.

the dimensionless parameter $p$. Our results show that the anisotropy can be enhanced by the boundary slip.

Using the FPD method, we verify the hydrodynamic reciprocal relation (33) and the angular dependence of the cross-coupling coefficients in Eq. (36) for the fluid-particle system illustrated in Fig. 2. The distance between the two walls is $H=2$, the major axis of the particle is $a=0.25$, and the aspect ratio is $e=0.5$. A diffuse fluid-solid interface with interfacial thickness $\xi=0.01$ is used to account for the fluid-particle coupling, with the boundary slip treated by the approach introduced in Sec. III B. The slip length $l_{s}$ varies from 0.03 to 0.1 . The no-slip condition is applied at the two walls. In case 1 , the particle rotates with constant angular velocity $\omega=-1$ and the walls are fixed. The integrated stress $S(\theta)$ at the upper wall is measured as a function of the instantaneous particle orientation $\theta$. In case 2 , the two walls move in the $\pm \hat{x}$ 

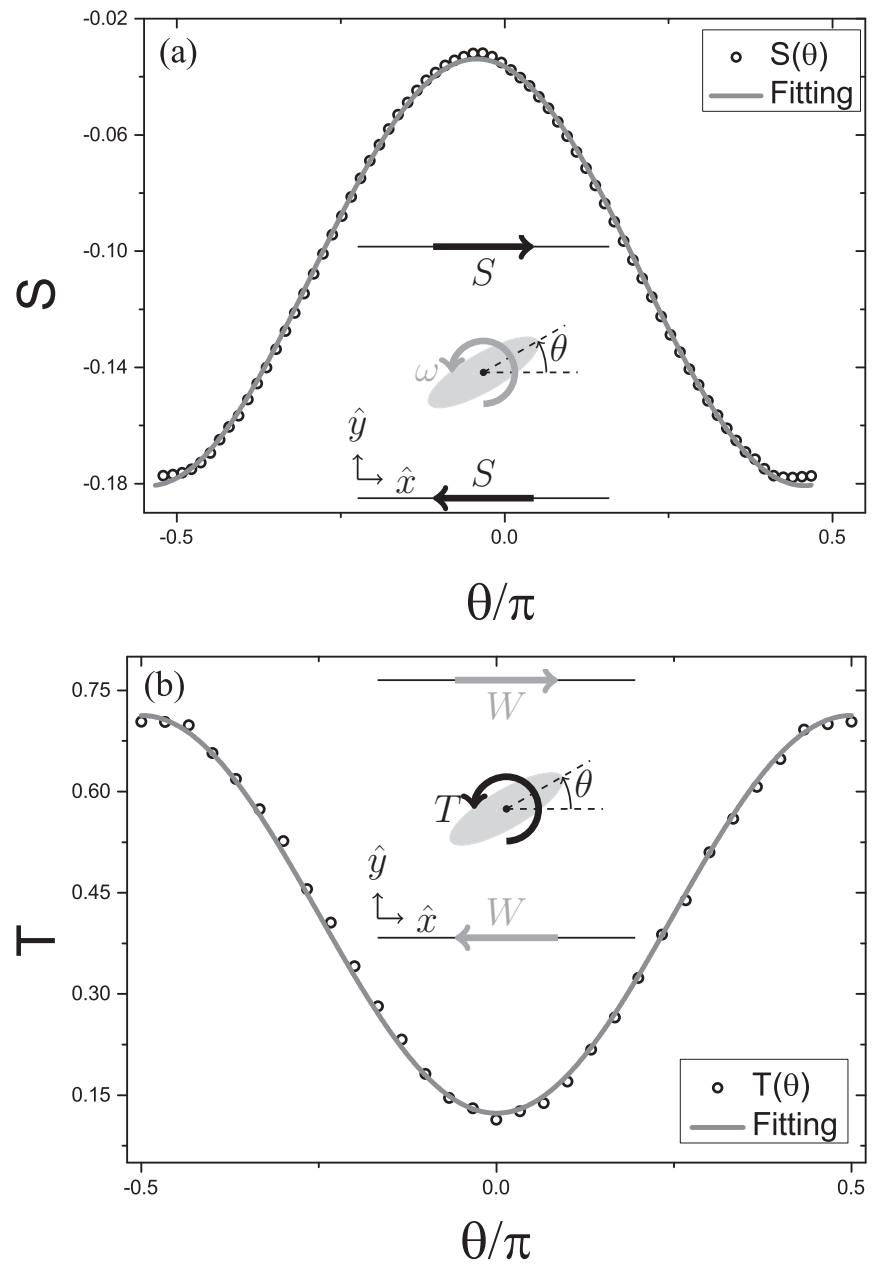

FIG. 4. Orientational dependence of the generalized forces, obtained for the numerical verification of the hydrodynamic reciprocal relations. (a) The integrated stress $S$ (by the upper wall on the fluid) plotted as a function of the particle orientation $\theta$ in case 1 . (b) The torque $T$ (by the particle on the fluid) plotted as a function of the particle orientation $\theta$ in case 2 . The symbols represent the results from the FPD simulations; the solid lines are the fitting curves according to Eq. (36). The insets in (a) and (b) illustrate case 1 and case 2, respectively. All the parameter values listed in the caption to Fig. 3 are used here.

directions with constant speed $W=2$ and the particle is fixed. The torque $T(\theta)$ is measured as a function of the particle orientation $\theta$. [Each data point is obtained in a steady-state flow generated for a fixed value of $\theta$.] For $l_{s}=5 \xi$, Figs. 3(a) and $3(\mathrm{~b})$ show the snapshots of the velocity field (vectors) and the particle (level curve of $\phi=0.5$ ) in case 1 and case 2 . Figures 4(a) and 4(b) show the corresponding results for $S(\theta)$ in case 1 and $T(\theta)$ in case 2, both in satisfactory agreement with the angular dependence in Eq. (36). A retardation in the response of $S$ to $\theta$ is observed in Fig. 4(a) as a small phase shift. This is attributed to the small but nonzero inertial effect. For different slip lengths, the dimensionless parameters $p_{S}$ and $p_{T}$ can be obtained by fitting $S(\theta)$ and $T(\theta)$, respectively, using the angular dependence in Eq. (36). As shown in Fig. 5, $p_{S}$ and $p_{T}$ satisfy the reciprocal symmetry fairly well (within $\sim 3 \%$ ). It is seen that the anisotropy parameter $p$ increases with

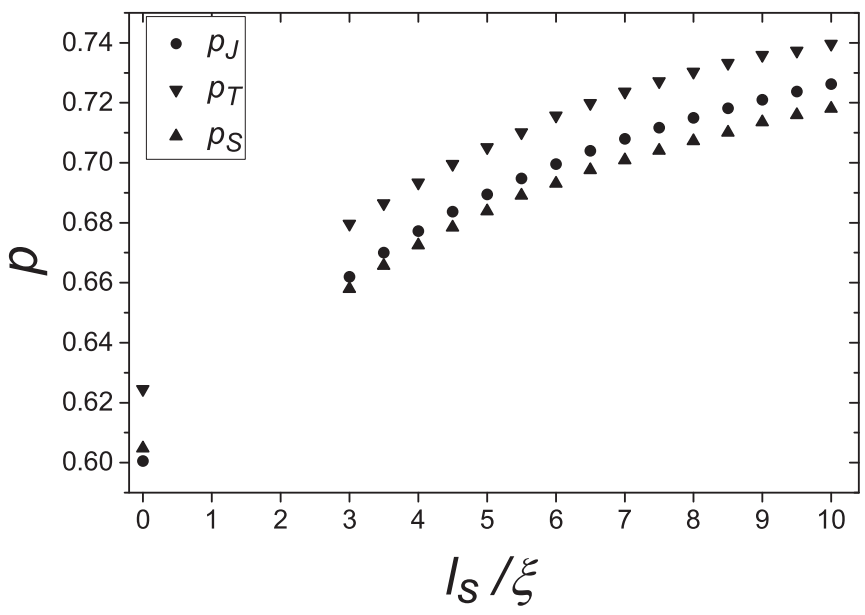

FIG. 5. The anisotropy parameter $p$ plotted as a function of the slip length $l_{s}$ at the particle surface. The major axis of the elliptical particle is $a=0.25$, and the aspect ratio is $e=0.5$. The fluid-solid interfacial thickness is $\xi=0.01$, and the slip length $l_{s}$ varies from $3 \xi$ to $10 \xi$. Here $p_{S}(\mathbf{\Delta})$ and $p_{T}(\boldsymbol{\nabla})$ are obtained through the numerical verification of the hydrodynamic reciprocal relations: $p_{S}$ is determined by fitting $S(\theta)$ and $p_{T}$ is determined by fitting $T(\theta)$ according to Eq. (36). In addition, $p_{J}(\bullet)$ is obtained from the FPD simulation of the Jeffery orbit: $p_{J}$ is determined by fitting the angular velocity $\omega(\theta)$ (shown in Fig. 6) according to Eq. (37). Note that in the limit of no slip $\left(l_{s}=0\right)$, the values of $p$ agree with Jeffery's result $p=0.6$ from Eq. (35) (within $\sim 3 \%$ ).

the increasing slip length at the particle surface. This means that the effective anisotropy of the particle can be enhanced by the boundary slip, i.e., the effective anisotropy is jointly determined by the geometric shape and the degree of boundary slip. In Sec. V, the anisotropy parameter $p$ will be discussed in relation to the cross coupling in nematic liquid crystals.

\section{B. Jeffery orbit}

In Jeffery's original work, the flow was assumed to be inertialess and the far-field components of distortion and rotation of the fluid were given. The torque by the fluid on the particle was calculated for no-slip ellipsoidal particles, and the equation of motion for the inertialess particle was obtained from the condition that the torque on the particle must vanish at every instant [5]. In our two-dimensional fluid-particle system, this condition gives

$$
\omega=\frac{\dot{\gamma}}{2}(p \cos 2 \theta-1)
$$

for $T$ expressed in Eq. (34). For no-slip elliptical particles, Jeffery gave $p=\left(1-e^{2}\right) /\left(1+e^{2}\right)$ and the solution

$$
\tan \theta=e \tan \left(\frac{\dot{\gamma} e}{1+e^{2}} t+C\right),
$$

with $C$ being a constant, for Eq. (37). It is worth emphasizing that Eq. (37) is applicable to slippery particles as well provided that $p$ is jointly determined by the geometric shape and the degree of boundary slip. A periodic solution can be obtained in the same form as in Eq. (38) but with an effective aspect ratio $e_{\text {eff }}=\sqrt{(1-p) /(1+p)}[7,20]$. If $p \geqslant 1$, however, a 

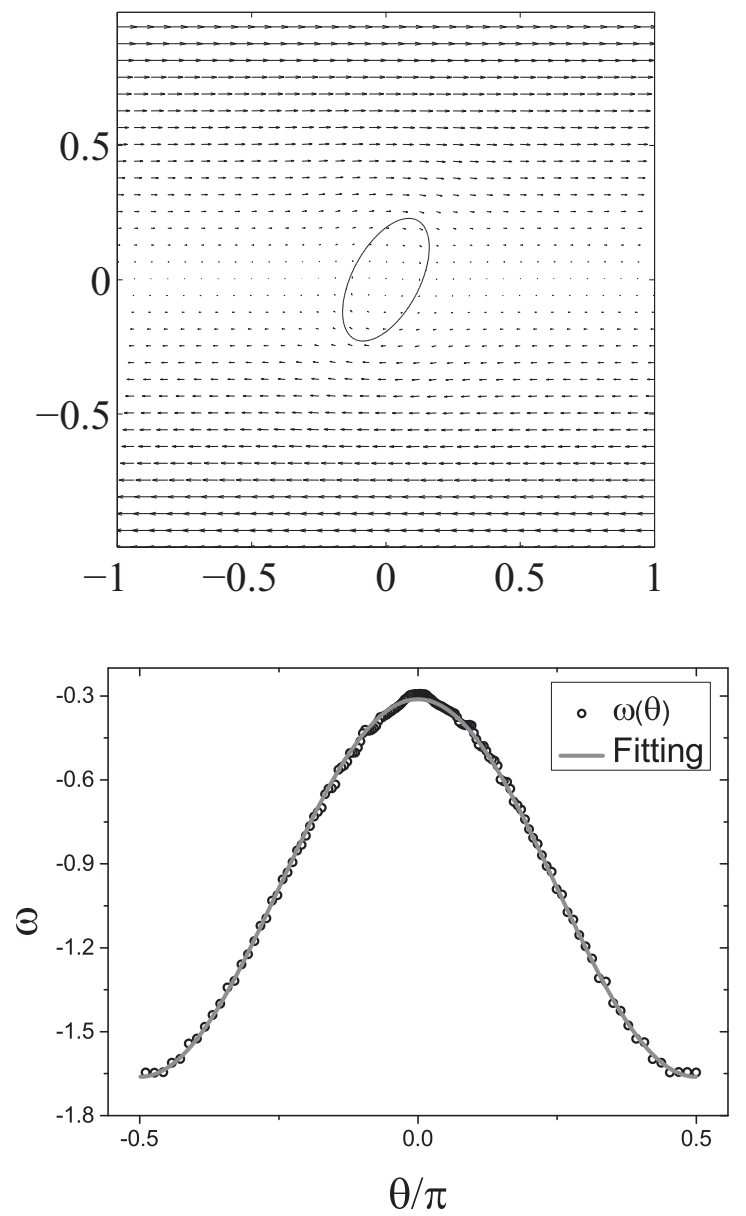

FIG. 6. The FPD simulation results for the Jeffery orbit of a slippery elliptical particle in a simple shear flow. The fluid-particle system measures $2 \times 2$, the shear rate is $\dot{\gamma}=2 \mathrm{~W} / H=2$, the major axis of the particle is $a=0.25$, and the aspect ratio is $e=0.5$. The fluid-solid interfacial thickness is $\xi=0.01$, and the slip length is $l_{s}=5 \xi$. (a) A snapshot of the velocity field (arrows) and the particle (level curve of $\phi=0.5$ ). (b) The angular velocity $\omega$ plotted as a function of the particle orientation $\theta$. The symbols represent the simulation results; the solid line is the fitting curve according to Eq. (37).

preferred orientation (shear alignment) is realized at $\cos 2 \theta=$ $1 / p$. To the best of our knowledge, there has been no report on the study of the Jeffery orbit with boundary slip. Below we numerically study the slippery Jeffery orbit to further demonstrate the effect of boundary slip on the anisotropy parameter $p$. We obtain results in quantitative agreement with those for $p_{S}$ and $p_{T}$ in Sec. IV A.

We use the same parameters as in Sec. IV A. The slip length $l_{s}$ varies from 0.03 to 0.1 . The no-slip condition is applied at the two walls. The two walls move in the $\pm x$ directions with constant speed $W=2$ (with $2 W / H=2$ ) and the particle rotates according to Eq. (37). For different slip lengths, the angular velocity $\omega(\theta)$ is recorded as a function of the instantaneous particle orientation $\theta$. The anisotropy parameter $p$ and the shear rate $\dot{\gamma}$ can be obtained by fitting $\omega(\theta)$ using Eq. (37). For $l_{s}=5 \xi$, Fig. 6(a) shows the snapshot of the flow field (vectors) and the particle (level curve of

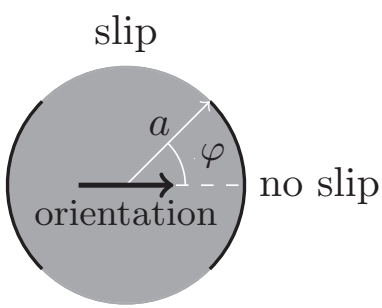

FIG. 7. A schematic illustration of a two-dimensional circular patchy particle. The particle surface is patterned with four sections, two with no-slip boundary condition and the other two with Navier boundary condition. The four sections are arranged in such a way that the patchy particle possesses the same symmetry as an ellipse. The diameter connecting the centers of two no-slip sections is used to denote the particle orientation. The particle radius is $a$, and $2 \varphi$ is the angular width of a no-slip section. The fraction covered by the no-slip sections is $c \equiv 2 \varphi / \pi$.

$\phi=0.5)$. Figure $6(\mathrm{~b})$ shows $\omega(\theta)$ in satisfactory agreement with Eq. (37). For $l_{s}$ varying from 0.03 to 0.1 , data fitting gives $\dot{\gamma}=1.9681 \pm 0.0002$, which is very close to $2 W / H=2$. The anisotropy parameter $p$ determined by fitting $\omega(\theta)$, denoted by $p_{J}$, is plotted in Fig. 5 for comparison with $p_{S}$ and $p_{T}$. It is seen that the three values of $p$, determined from three distinct cases, are very close (within $\sim 3 \%$ ).

Finally, we estimate the quantitative effect of slip on the effective aspect ratio $e_{\text {eff }}=\sqrt{(1-p) /(1+p)}$. For a particle with major axis $a=0.25$, aspect ratio $e=0.5$, and slip length $l_{s}=0.03$, the measured effective anisotropy is $p=0.66654$ (from an average of $p_{S}, p_{T}$, and $p_{J}$ ), which gives $e_{\mathrm{eff}}=0.44732$. Since the slip length is defined as an extrapolation length into the solid, we propose that the effective shape of the particle has the major and minor axes given by $a-\alpha l_{s}$ and $b-\alpha l_{s}$, in which $\alpha$ is a constant $\simeq 1$. From $e_{\text {eff }}=\left(b-\alpha l_{s}\right) /\left(a-\alpha l_{s}\right)$, we find $\alpha=0.794$, which is $\simeq 1$, as expected.

\section{Jeffery orbit of a circular patchy particle}

We have shown that the boundary slip can change the effective anisotropy of an elliptical particle. Now we turn to the joint effect of surface patching and boundary slip on the effective anisotropy of a circular particle. We focus on the dynamics of a two-dimensional circular patchy particle in a simple shear flow. As shown in Fig. 7, the particle surface is patterned with four sections, two with no-slip boundary condition and the other two with Navier boundary condition. The four sections are arranged in such a way that the patchy particle possesses the same symmetry as an ellipse. The diameter connecting the centers of two no-slip sections is used to denote the particle orientation. The particle radius is $a$, and $2 \varphi$ is the angular width of a no-slip section. We use $c \equiv 2 \varphi / \pi$ to denote the fraction covered by the no-slip sections.

Consider that this patchy particle is subject to a simple shear flow generated by two moving walls as in Fig. 2. As stated in Sec. IV A, this simple shear flow can be decomposed into an isotropic rotation field and an anisotropic distortion field. Furthermore, the torque exerted by the particle on the fluid is a sum of two contributions: The first is from the rotation 

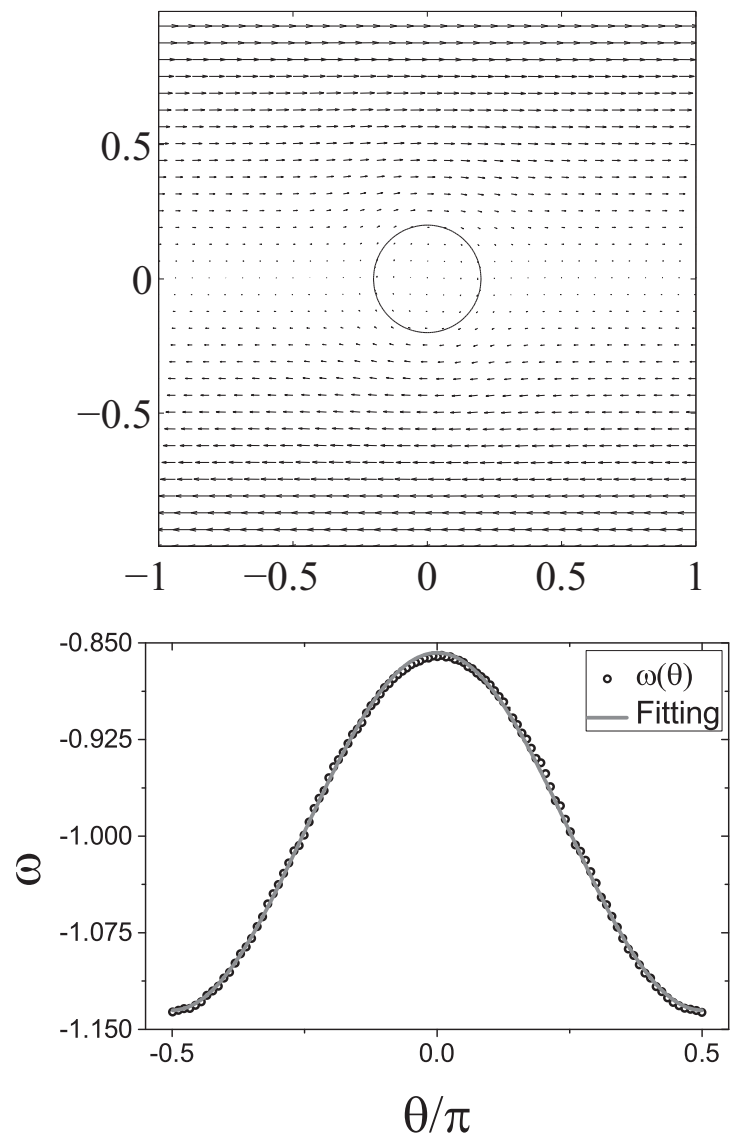

FIG. 8. The FPD simulation results for the Jeffery orbit of a circular patchy particle in a simple shear flow. The fluid-particle system measures $2 \times 2$, the shear rate is $\dot{\gamma}=2 \mathrm{~W} / H=2$, the particle radius is $a=0.2$, the fluid-solid interfacial thickness is $\xi=0.01$, the no-slip fraction is $c=0.5$, and the slip length in the slip sections is $l_{s}=0.15$. (a) A snapshot of the velocity field (arrows) and the particle (level curve of $\phi=0.5$ ). (b) The angular velocity $\omega$ plotted as a function of the particle orientation $\theta$. The symbols represent the simulation results; the solid line is the fitting curve according to Eq. (37).

of the particle relative to the fluid and the second is from the mismatch between the particle orientation and the principal axes of the distortion field. As the patchy particle possesses the same symmetry as an ellipse, the torque by the particle on the fluid still scales as

$$
T \propto\left(\omega+\frac{\dot{\gamma}}{2}\right)-\frac{\dot{\gamma}}{2} p \cos 2 \theta,
$$

which is identical to Eq. (34). The equation of motion for the inertialess particle is given by $T=0$, which leads to Eq. (37) once again: The orientational motion of the circular patchy particle follows the Jeffery orbit. Below we study the joint effect of surface patching and boundary slip on the effective anisotropy measured by $p$. Note that $p$ vanishes at $c=0$ and $c=1$ due to circular symmetry. It is therefore expected that for a given slip length in the slippery sections, $p$ will be maximized at $c$ somewhere between 0 and 1 .

As shown in Fig. 2, the circular patchy particle (with an orientation denoted by the diameter connecting the centers of two no-slip sections) is placed at the center and subject

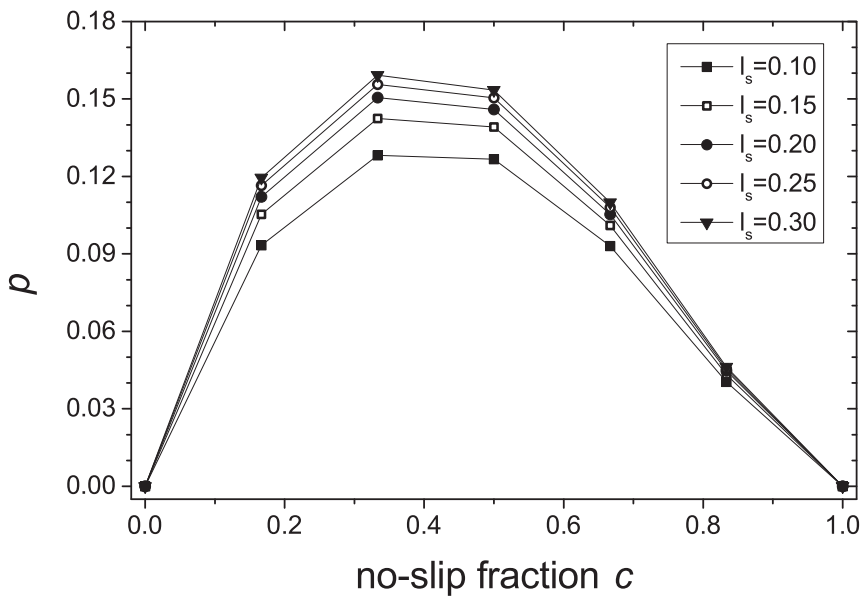

FIG. 9. The anisotropy parameter $p$ of a circular patchy particle from the FPD simulations, plotted as a function of the no-slip fraction $c$, for different values of the slip length in the slip sections $l_{s}$. The particle radius is $a=0.2$, and the fluid-solid interfacial thickness is $\xi=0.01$. Simulations are carried out for the no-slip fraction $c=1 / 6$, $1 / 3,1 / 2,2 / 3$, and $5 / 6$, and the slip length in the slip sections $l_{s}=0.10$ $(\boldsymbol{\square}), 0.15(\square), 0.20(\bullet), 0.25(\circ)$, and $0.30(\nabla)$.

to a simple shear flow generated by two moving walls. We use $H=2$ for the distance between the two walls, $\xi=0.01$ for the interfacial thickness, $a=0.2$ for the particle radius, and $W=2$ for the wall speed. Simulations are carried out for different values of the no-slip fraction $c$ and the slip length $l_{s}$. This is to verify whether the orientational motion satisfies Eq. (37) and to measure the effects of $c$ and $l_{s}$ on $p$. For $c=0.5$ and $l_{s}=0.15$, Fig. 8(a) shows the snapshot of the flow field (vectors) and the particle (level curve of $\phi=0.5$ ), and Fig. 8(b) shows the angular velocity $\omega(\theta)$ as a function of the instantaneous particle orientation $\theta$, which is in good agreement with Eq. (37). With the anisotropy parameter $p$ determined by fitting $\omega(\theta)$, we plot in Fig. 9 the effects of $c$ and $l_{s}$ on $p$. It is seen that when the slip length $l_{s}$ is fixed, $p$ shows a nonmonotonic dependence on $c$, with a maximum at $c \approx 0.4$. When the no-slip fraction $c$ is fixed, $p$ always increases with the increasing $l_{s}$. However, this increase slows down for large $l_{s}$, implying a possible saturation of $p$ at $l_{s} \rightarrow \infty$.

\section{DISCUSSION: ANALOGY BETWEEN THE FLUID-PARTICLE SYSTEM AND A UNIT OF NEMATIC LIQUID CRYSTAL}

An analogy can be readily made between the fluid-particle system shown in Fig. 2 and a unit of nematic liquid crystal (NLC). For incompressible isothermal NLCs described by the Leslie-Ericksen theory [93-95], Parodi used the Onsager reciprocal relations to derive a celebrated symmetry relation, called the Parodi relation [96], for the phenomenological coefficients in the constitutive equation for the stress tensor. Employing the Parodi relation, we find that a reciprocal relation similar to Eq. (36) can be derived for a unit of NLC. This demonstrates an analogy between the fluid-particle system in Fig. 2 and a unit of NLC. In fact, the connection between the Leslie-Ericksen theory and the Jeffery orbit has been long recognized [27]. The equation of Jeffery orbit has 
been frequently used in NLC studies for the coupling between molecular rotation and fluid flow [25-30].

The Leslie-Ericksen theory gives the dissipative stress tensor $\stackrel{\leftrightarrow}{\sigma}$ and the torque density by the director on the fluid $\vec{\Gamma}$ as

$$
\begin{gathered}
\stackrel{\leftrightarrow}{\sigma}=\alpha_{1}(\vec{n} \vec{n}: \overleftrightarrow{A}) \vec{n} \vec{n}+\alpha_{2} \vec{n} \vec{N}+\alpha_{3} \vec{N} \vec{n}+\alpha_{4} \overleftrightarrow{A} \\
+\alpha_{5} \vec{n}(\vec{n} \cdot \overleftrightarrow{A})+\alpha_{6}(\vec{n} \cdot \overleftrightarrow{A}) \vec{n}, \\
\vec{\Gamma}=\vec{n} \times\left(\gamma_{1} \vec{N}+\gamma_{2} \overleftrightarrow{A} \cdot \vec{n}\right),
\end{gathered}
$$

where $\alpha_{i}(i=1, \ldots, 6)$ are phenomenological parameters, $\vec{n}$ is the director, $\stackrel{\leftrightarrow}{A} \equiv \frac{1}{2}\left(\vec{\nabla} \vec{v}+\vec{\nabla} \vec{v}^{T}\right)$ is the rate-of-strain tensor of flow, and $\vec{N} \equiv \dot{\vec{n}}-\vec{v} \times \vec{n}=(\vec{\omega}-\vec{v}) \times \vec{n}$ is the velocity of the director relative to the fluid, with $\vec{\omega}$ being the angular velocity of the director and $\vec{v} \equiv \frac{1}{2} \vec{\nabla} \times \vec{v}$ being the angular velocity of the fluid. In addition, $\gamma_{1}$ and $\gamma_{2}$ are given by $\gamma_{1}=\alpha_{3}-\alpha_{2}$ and $\gamma_{2}=\alpha_{6}-\alpha_{5}$. Physically, the two contributions to $\vec{\Gamma}$ in Eq. (40) correspond to the two contributions to $T$ in Eq. (34) [27]. Moreover, only five of the six $\alpha_{i}(i=1, \ldots, 6)$ parameters are independent because of the Parodi relation

$$
\alpha_{2}+\alpha_{3}=\alpha_{6}-\alpha_{5},
$$

which results from the Onsager reciprocal relations [96].

Consider an NLC system which is actually twodimensional. The director is denoted by a unit vector $\vec{n} \equiv$ $(\cos \theta, \sin \theta, 0)$. If the velocity field is given by a simple shear flow with $\partial_{y} v_{x}=\dot{\gamma}$ and the angular velocity of the director is $\omega \hat{z}$, then we have the following components:

$$
\begin{aligned}
\sigma_{y x}= & \alpha_{1} \cos ^{2} \theta \sin ^{2} \theta \dot{\gamma}-\alpha_{2} \sin ^{2} \theta\left(\omega+\frac{\dot{\gamma}}{2}\right) \\
& +\alpha_{3} \cos ^{2} \theta\left(\omega+\frac{\dot{\gamma}}{2}\right)+\alpha_{4} \frac{\dot{\gamma}}{2}+\alpha_{5} \sin ^{2} \theta \frac{\dot{\gamma}}{2} \\
& +\alpha_{6} \cos ^{2} \theta \frac{\dot{\gamma}}{2}, \\
\sigma_{x y}= & \alpha_{1} \cos ^{2} \theta \sin ^{2} \theta \dot{\gamma}+\alpha_{2} \cos ^{2} \theta\left(\omega+\frac{\dot{\gamma}}{2}\right) \\
& -\alpha_{3} \sin ^{2} \theta\left(\omega+\frac{\dot{\gamma}}{2}\right)+\alpha_{4} \frac{\dot{\gamma}}{2}+\alpha_{5} \cos ^{2} \theta \frac{\dot{\gamma}}{2} \\
& +\alpha_{6} \sin ^{2} \theta \frac{\dot{\gamma}}{2}, \\
\Gamma_{z}= & \gamma_{1}\left(\omega+\frac{\dot{\gamma}}{2}\right)+\gamma_{2} \cos 2 \theta \frac{\dot{\gamma}}{2},
\end{aligned}
$$

for the dissipative stress tensor and torque density according to Eqs. (39) and (40), with $\sigma_{x y}-\sigma_{y x}=-\Gamma_{z}$.

The rate of free-energy dissipation is given by

$$
D=\iint d x d y\left(\sigma_{i j} \partial_{i} v_{j}+\Gamma_{z} \omega\right)=\iint d x d y\left(\dot{\gamma} \sigma_{y x}+\Gamma_{z} \omega\right) .
$$

For the NLC system considered here, the two rates are the shear rate $\dot{\gamma}$ and the director angular velocity $\omega$, with $\sigma_{y x}$ and $\Gamma_{z}$ being the corresponding conjugate dissipative forces. A linear relation between the rates and the forces can be expressed as

$$
\left[\begin{array}{c}
\sigma_{y x} \\
\Gamma_{z}
\end{array}\right]=\left[\begin{array}{ll}
\xi_{11} & \xi_{12} \\
\xi_{21} & \xi_{22}
\end{array}\right]\left[\begin{array}{l}
\dot{\gamma} \\
\omega
\end{array}\right]
$$

with the reciprocal relation

$$
\xi_{12}=\xi_{21} \text {. }
$$

From Eq. (42) and the Parodi relation (41), we have

$$
\xi_{12}=\xi_{21}=\frac{1}{2}\left(\gamma_{1}+\gamma_{2} \cos 2 \theta\right) .
$$

Consider a rectangular unit of NLC in which the shear rate $\partial_{y} v_{x}=\dot{\gamma}$ and the angular velocity of the director $\omega$ are homogeneous. The corresponding rate of free-energy dissipation in Eq. (43) can be expressed as

$$
D=\left(\int d y \dot{\gamma}\right)\left(\int d x \sigma_{y x}\right)+\omega \iint d y d x \Gamma_{z} .
$$

A comparison can be made with the fluid-particle system in Fig. 2, for which the rate of dissipation is given by Eq. (31). It is clear that $\int d y \dot{\gamma}$ in the NLC unit corresponds to the rate $2 W$ in Eq. (31), the angular velocity of the director $\omega$ corresponds to that of the particle, $\int d x \sigma_{y x}$ in the NLC unit corresponds to the integrated stress $S$, and $\iint d y d x \Gamma_{z}$ corresponds to the torque by the particle on the fluid $T$. In this regard, the dissipation rates (31) and (46) are identical. A comparison of the reciprocal relations (45) and (36) leads to

$$
p=-\frac{\gamma_{2}}{\gamma_{1}} \text {. }
$$

For the one-particle system studied in the present work, we have $0<p<1$, which means that the particle undergoes periodic tumbling motion in viscous shear flow. It is interesting to note that for many nematic liquid crystals, we have $-\gamma_{2} / \gamma_{1}>$ 1 , which results in shear alignment. It might be possible to achieve shear alignment in the one-particle system through a combination of particle geometry and surface patching.

\section{CONCLUDING REMARKS}

Based on Onsager's variational principle, we have shown that the Navier slip boundary condition preserves the hydrodynamic reciprocal relations. By incorporating the Navier slip boundary condition into the FPD method, we have investigated the effect of boundary slip on the motion of an anisotropic particle in a simple shear flow. We have carried out numerical simulations for a fluid-particle system with a slippery elliptical particle. This system exhibits the cross coupling between the rotational torque and the shear stress. Theoretical analysis gives the angular dependence of the cross-coupling coefficients and the dynamic equation of the particle in a simple shear flow-the Jeffery orbit. The effective anisotropy of the particle is measured by a dimensionless parameter $p$, whose value can be numerically determined. We have demonstrated that the anisotropy parameter $p$ increases with the increasing slip length at the particle surface. This means the cross coupling between the rotational torque and the shear stress is enhanced by the boundary slip. In addition, we have carried out numerical simulations for a circular patchy particle whose surface is patterned with sections of no slip and Navier slip. Possessing the same symmetry as 
the elliptical particle, the circular patchy particle is effectively anisotropic and undergoes the Jeffery orbit in a simple shear flow. By numerically measuring the anisotropy parameter $p$, we have demonstrated the joint effect of surface patching and boundary slip on the effective anisotropy. Finally, there is a brief discussion on the connection between the fluid-particle system and the nematic liquid crystal.

Although our simulations are carried out in two dimensions, we believe that the qualitative conclusion that the boundary slip at particle surface will enhance the effective anisotropy of the particle still holds in three dimensions. However, quantitative verification requires numerical simulations that are currently beyond our capability. Furthermore, the data analysis will be more complicated because the three-dimensional Jeffery orbit is beyond the simple description by Eq. (37).

Our results have demonstrated the effect of boundary slip on the effective anisotropy of the solid particle in viscous shear flow, which is reflected in the cross coupling between the rotational torque and the shear stress, the hydrodynamic reciprocal relations, and the Jeffery orbit. Physically, the Jeffery orbit is directly employed in the kinetic models for liquid crystal dynamics [25-30]. The results presented in this work indicate that rheological properties can be modified by engineering the surface of dispersed particles. We note that our results are obtained for one single particle, whereas hydrodynamic interactions are significant when dispersed particles in colloids form certain structures [97-99]. By engineering the surface of patchy particles, novel structures may be expected [100-103]. Our results for patchy particles indicate that novel dynamic properties may be expected as well by engineering the particle surface.

\section{ACKNOWLEDGMENTS}

This work is supported by Hong Kong RGC Grant No. HKUST604013 and Project No. SRFI11SC02 of HKUST.
T. Qian thanks C. Liu of Penn State University and Q. Wang of University of South Carolina for helpful discussion.

\section{APPENDIX: THE FLUID PARTICLE DYNAMICS METHOD}

Here we give a brief description of the FPD algorithm. For simplicity, we consider one single particle. More details can be found in Ref. [76]. The initial condition gives the initial values of center-of-mass position $\vec{R}_{p}$ and orientation $\vec{d}_{p}$ of the particle as well as the velocity field $\vec{v}(\vec{r})$. The time evolution from the $n$-th to the $(n+1)$-th time instant is computed as follows.

(1) Generate the composition field $\phi^{n}(\vec{r})$ according to Eq. (24) and then the viscosity distribution $\eta^{n}(\phi)$.

(2) Calculate the velocities $\vec{V}_{p}^{n}$ and $\vec{\omega}_{p}^{n}$ of the particle using $\vec{v}^{n}(\vec{r})$ and a weight function $w(\vec{r})$ with $w=1$ in the solid and $w=0$ in the fluid.

(3) Update the center-of-mass position and orientation of the particle using $\vec{V}_{p}^{n}$ and $\vec{\omega}_{p}^{n}$.

(4) Solve the Navier-Stokes equation to update the velocity field from $\vec{v}^{n}(\vec{r})$ to $\vec{v}^{n+1}(\vec{r})$.

Note that the choice of weight function $w(\vec{r})$ may slightly influence the results. Because a larger viscosity ensures a better approximation to the rigid body motion, the results are expected to be more accurate if $w(\vec{r})=1$ is confined to the region with sufficiently large viscosity. H. Tanaka and T. Araki used $w(\vec{r})=\phi(\vec{r})$ [76], and we use the relative viscosity [104]

$$
w(\vec{r})= \begin{cases}\left(\eta-\eta_{l}\right) /\left(\eta_{s}-\eta_{l}\right) & \text { if } \quad \eta \geqslant \eta_{l} \\ 0 & \text { if } \quad \eta<\eta_{l}\end{cases}
$$

where $\eta<\eta_{l}$ occurs in the interfacial region when slip is introduced.
[1] J. Happel and H. Brenner, Low Reynolds Number Hydrodynamics: With Special Applications to Particulate Media, Vol. 1 (Springer, Berlin, 1983).

[2] D. J. Jeffrey and A. Acrivos, AIChE J. 22, 417 (1976).

[3] J. J. Stickel and R. L. Powell, Annu. Rev. Fluid Mech. 37, 129 (2005).

[4] H. Brenner, Adv. Chem. Eng. 6, 287 (1966).

[5] G. B. Jeffery, Proc. R. Soc. Lond. Ser. A 102, 161 (1922).

[6] G. I. Taylor, Proc. R. Soc. Lond. A 103, 58 (1923).

[7] F. P. Bretherton, J. Fluid Mech. 14, 284 (1962).

[8] B. J. Trevelyan and S. G. Mason, J. Colloid Sci. 6, 354 (1951).

[9] O. L. Forgacs and S. G. Mason, J. Colloid Sci. 14, 457 (1959).

[10] H. L. Goldsmith and S. G. Mason, J. Fluid Mech. 12, 88 (1962).

[11] M. S. Ingber and L. A. Mondy, J. Rheol. (NY) 38, 1829 (1994).

[12] C. A. Stover and C. Cohen, Rheol. Acta 29, 192 (1990).

[13] P. Skjetne, R. F. Ross, and D. J. Klingenberg, J. Chem. Phys. 107, 2108 (1997).

[14] C. Pozrikidis, J. Fluid Mech. 541, 105 (2005).

[15] E.-J. Ding and C. K. Aidun, J. Fluid Mech. 423, 317 (2000).
[16] D. Qi and L.-S. Luo, J. Fluid Mech. 477, 201 (2003).

[17] G. Subramanian and D. L. Koch, J. Fluid Mech. 535, 383 (2005).

[18] Z. Yu, N. Phan-Thien, and R. I. Tanner, Phys. Rev. E 76, 026310 (2007).

[19] F. Lundell and A. Carlsson, Phys. Rev. E 81, 016323 (2010).

[20] H. L. Goldsmith and S. G. Mason, J. Colloid Sci. 17, 448 (1962).

[21] R. G. Cox and S. G. Mason, Annu. Rev. Fluid Mech. 3, 291 (1971).

[22] A. T. Chwang, J. Fluid Mech. 72, 17 (1975).

[23] D. Vincenzi, J. Fluid Mech. 719, 465 (2013).

[24] C. Ferrari, B. Kaoui, V. S. L’vov, I. Procaccia, O. Rudenko, J. H. M. ten Thije Boonkkamp, and F. Toschi, Phys. Rev. E 86, 016302 (2012).

[25] N. Kuzuu and M. Doi, J. Phys. Soc. Jpn. 52, 3486 (1983).

[26] R. G. Larson and L. A. Archer, Liq. Cryst. 19, 883 (1995).

[27] C. V. Chaubal and L. G. Leal, J. Non-Newtonian Fluid Mech. 82, 25 (1999). 
[28] Q. Wang, J. Chem. Phys. 116, 9120 (2002).

[29] M. G. Forest, R. Zhou, and Q. Wang, Phys. Rev. E 66, 031712 (2002).

[30] M. G. Forest, Q. Wang, and R. Zhou, Soft Matter 9, 5207 (2013).

[31] T. Gao and H. H. Hu, J. Comput. Phys. 228, 2132 (2009).

[32] T. Gao, H. H. Hu, and P. P. Castañeda, J. Fluid Mech. 687, 209 (2011).

[33] T. Gao, H. H. Hu, and P. P. Castañeda, Phys. Rev. Lett. 108, 058302 (2012).

[34] J. Beaucourt, F. Rioual, T. Séon, T. Biben, and C. Misbah, Phys. Rev. E 69, 011906 (2004).

[35] H. Zhao and E. S. G. Shaqfeh, J. Fluid Mech. 674, 578 (2011).

[36] B. Kaoui, T. Krüger, and J. Harting, Soft Matter 8, 9246 (2012).

[37] D. Saintillan and M. J. Shelley, Phys. Rev. Lett. 100, 178103 (2008).

[38] T. Kaya and H. Koser, Phys. Rev. Lett. 103, 138103 (2009).

[39] P. Legendre and M. J. Fortin, Vegetatio 80, 107 (1989).

[40] J. W. Costerton, P. S. Stewart, and E. P. Greenberg, Science 284, 1318 (1999).

[41] J. W. Costerton, Z. Lewandowski, D. E. Caldwell, D. R. Korber, and H. M. Lappin-Scott, Annu. Rev. Microbiol. 49, 711 (1995).

[42] J. S. Guasto, R. Rusconi, and R. Stocker, Annu. Rev. Fluid Mech. 44, 373 (2012).

[43] W. M. Durham, E. Climent, and R. Stocker, Phys. Rev. Lett. 106, 238102 (2011).

[44] E. Lushi, R. E. Goldstein, and M. J. Shelley, Phys. Rev. E 86, 040902 (2012).

[45] A. M. Ardekani and E. Gore, Phys. Rev. E 85, 056309 (2012).

[46] C. Zhan, G. Sardina, E. Lushi, and L. Brandt, J. Fluid Mech. 739, 22 (2014).

[47] G. K. Batchelor, An Introduction to Fluid Dynamics (Cambridge University Press, Cambridge, UK, 2000).

[48] E. M. Purcell, Am. J. Phys. 45, 3 (1977).

[49] S. Kim and S. J. Karrila, Microhydrodynamics: Principles and Selected Applications (Butterworth-Heinemann, London, 1991).

[50] L. G. Leal, Advanced Transport Phenomena: Fluid Mechanics and Convective Transport Processes (Cambridge University Press, Cambridge, UK, 2007).

[51] C. Pozrikidis, Boundary Integral and Singularity Methods for Linearized Viscous Flow (Cambridge University Press, Cambridge, UK, 1992).

[52] W. E. Olmstead, Acta Mech. 21, 289 (1975).

[53] H. A. Lorentz, Zittingsverslag van de Koninklijke Akademie van Wetenschappen te Amsterdam 5, 168 (1896).

[54] L. Onsager, Phys. Rev. 37, 405 (1931).

[55] L. Onsager, Phys. Rev. 38, 2265 (1931).

[56] R. J. Phillips, J. Fluid Mech. 315, 345 (1996).

[57] H. A. Stone and A. D. T. Samuel, Phys. Rev. Lett. 77, 4102 (1996).

[58] A. Nadim, H. Haj-Hariri, and A. Borhan, Part. Sci. Technol. 8, 191 (1990).

[59] H. Masoud and H. A. Stone, J. Fluid Mech. 741, R4 (2014).

[60] A. S. Khair, D. E. Posluszny, and L. M. Walker, Phys. Rev. E 85, 016320 (2012).

[61] K. Kamrin and H. A. Stone, Phys. Fluids 23, 031701 (2011).

[62] Y. Zhu and S. Granick, Phys. Rev. Lett. 88, 106102 (2002).
[63] E. Lauga, M. Brenner, and H. Stone, in Springer Handbook of Experimental Fluid Mechanics, edited by C. Tropea, A. L. Yarin, and J. F. Foss (Springer, Berlin, 2007), Chap. 19, pp. 1219-1240.

[64] W.-M. Zhang, G. Meng, and X. Wei, Microfluidics and Nanofluidics 13, 845 (2012).

[65] T. Qian, X.-P. Wang, and P. Sheng, Phys. Rev. E 68, 016306 (2003).

[66] J.-L. Barrat and L. Bocquet, Phys. Rev. Lett. 82, 4671 (1999).

[67] J. Baudry, E. Charlaix, A. Tonck, and D. Mazuyer, Langmuir 17, 5232 (2001).

[68] Y. Zhu and S. Granick, Phys. Rev. Lett. 87, 096105 (2001).

[69] C. L. M. H. Navier, Mémoires de l'Académie Royale des Sciences de l'Institut de France 6, 389 (1823).

[70] T. Qian, X.-P. Wang, and P. Sheng, J. Fluid Mech. 564, 333 (2006).

[71] H. Sun and C. Liu, Solid State Commun. 150, 990 (2010).

[72] J. F. Brady, R. J. Phillips, J. C. Lester, and G. Bossis, J. Fluid Mech. 195, 257 (1988).

[73] D. R. Foss and J. F. Brady, J. Fluid Mech. 407, 167 (2000).

[74] C. Reichhardt and C. J. Olson, Phys. Rev. Lett. 89, 078301 (2002).

[75] D. Sulsky and J. U. Brackbill, J. Comput. Phys. 96, 339 (1991).

[76] H. Tanaka and T. Araki, Phys. Rev. Lett. 85, 1338 (2000).

[77] H. Tanaka and T. Araki, Chem. Eng. Sci. 61, 2108 (2006).

[78] P.-G. de Gennes, Langmuir 18, 3413 (2002).

[79] D. Andrienko, B. Dünweg, and O. I. Vinogradova, J. Chem. Phys. 119, 13106 (2003).

[80] D. G. Miller, Chem. Rev. 60, 15 (1960).

[81] S. R. de Groot and P. Mazur, Non-Equilibrium Thermodynamics (Dover, London, 1984).

[82] J. W. Strutt, Proc. Lond. Math. Soc. 1, 357 (1871).

[83] M. Doi, J. Phys.: Condens. Matter 23, 284118 (2011).

[84] F. Osterle, Appl. Sci. Res. 49, 369 (1992).

[85] M. Doi, in Non-Equilibrium Soft Matter Physics, Vol. 4, edited by S. Komura and T. Ohta (World Scientific, Singapore, 2012), Chap. 1, pp. 1-35.

[86] M. Cieplak, J. Koplik, and J. R. Banavar, Phys. Rev. Lett. 86, 803 (2001).

[87] P. A. Thompson and S. M. Troian, Nature 389, 360 (1997).

[88] V. S. J. Craig, C. Neto, and D. R. M. Williams, Phys. Rev. Lett. 87, 054504 (2001).

[89] M. T. Matthews and J. M. Hill, Acta Mech. 191, 195 (2007).

[90] H. H. Hu, Int. J. Multiphase Flow 22, 335 (1996).

[91] G. Tryggvason, R. Scardovelli, and S. Zaleski, Direct Numerical Simulations of Gas-Liquid Multiphase Flows (Cambridge University Press, Cambridge, UK, 2011).

[92] E. J. Hinch, J. Fluid Mech. 54, 423 (1972).

[93] J. L. Ericksen, Arch. Ration. Mech. Anal. 4, 231 (1959).

[94] F. M. Leslie, Q. J. Mech. Appl. Math. 19, 357 (1966).

[95] F. M. Leslie, Arch. Ration. Mech. Anal. 28, 265 (1968).

[96] O. Parodi, J. Phys. 31, 581 (1970).

[97] H. Tanaka, Phys. Rev. E 59, 6842 (1999).

[98] H. Tanaka, J. Phys.: Condens. Matter 12, R207 (2000).

[99] C. P. Royall, S. R. Williams, T. Ohtsuka, and H. Tanaka, Nat. Mater. 7, 556 (2008).

[100] Z. Zhang and S. C. Glotzer, Nano Lett. 4, 1407 (2004). 
[101] S. C. Glotzer and M. J. Solomon, Nat. Mater. 6, 557 (2007).

[102] J. P. K. Doye, A. A. Louis, I.-C. Lin, L. R. Allen, E. G. Noya, A. W. Wilber, H. C. Kok, and R. Lyus, Phys. Chem. Chem. Phys. 9, 2197 (2007).
[103] A. B. Pawar and I. Kretzschmar, Macromolec. Rapid Commun. 31, 150 (2010).

[104] L. Jibuti, Locomotion and Flow in Complex and Confined Fluids, Ph.D. thesis, Université de Grenoble, Grenoble, France, 2011. 